\title{
Fast-Neutron Activation of Long-Lived Nuclides in Natural $\mathrm{Pb}$
}

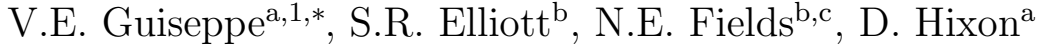 \\ ${ }^{a}$ University of South Dakota, Vermillion, SD 57069 \\ ${ }^{b}$ Los Alamos National Laboratory, Los Alamos, NM 87545 \\ ${ }^{c}$ University of Chicago, Chicago, IL 60637
}

\begin{abstract}
We measured the production of the long-lived nuclides ${ }^{207} \mathrm{Bi},{ }^{202} \mathrm{~Pb}$, and ${ }^{194} \mathrm{Hg}$ in a sample of natural $\mathrm{Pb}$ due to high-energy neutron interactions using a neutron beam at the Los Alamos Neutron Science Center. The activated sample was counted by a HPGe detector to measure the amount of radioactive nuclides present. These nuclides are critical in understanding potential backgrounds in low background experiments utilizing large amounts of $\mathrm{Pb}$ shielding due to cosmogenic neutron interactions in the $\mathrm{Pb}$ while residing on the Earth's surface. By scaling the LANSCE neutron flux to a cosmic neutron flux, we measure the sea level cosmic ray production rates of $8.0 \pm$ 1.3 atoms $/ \mathrm{kg} /$ day of ${ }^{194} \mathrm{Hg}, 120 \pm 25$ atoms $/ \mathrm{kg} /$ day ${ }^{202} \mathrm{~Pb}$, and $<0.17 \pm$ 0.04 atoms $/ \mathrm{kg} /$ day ${ }^{207} \mathrm{Bi}$.
\end{abstract}

Keywords: neutron activation, double-beta decay, lead, cosmic ray

\section{Introduction}

An observation of neutrinoless double-beta decay $(0 \nu \beta \beta)$ is critical to understanding the neutrino's absolute mass scale and particle-antiparticle nature $[1,2,3,4,5,6,7]$. The signature of this rare nuclear decay process would be the detection of a mono-energetic line within the the material containing an isotope subject to this decay mode. The potential success of these experiments relies on the ability to mitigate backgrounds that would mask the rare

\footnotetext{
*Corresponding author

Email address: guiseppe@sc.edu (V.E. Guiseppe)

${ }^{1}$ Present Address: University of South Carolina, Columbia, SC 29208
} 
signal of interest through a reduction of internal radioactive backgrounds and ability to shield the detectors from external sources of radioactivity. Trace levels of radioactive impurities from natural decay chain isotopes within the detector and shielding components have been the limiting backgrounds in previous experiments. The $\gamma$-ray emissions from internal and external backgrounds can overwhelm the potential $0 \nu \beta \beta$ signal by producing a continuum of energetic deposits inside the detector. Ongoing and future experiments continue to identify and reduce potential backgrounds to the signal of interest. A background level goal of 1 event/ton-year, however, is an ambitious improvement over the currently best achieved background level [8,9]. With the efforts to reduce the natural decay chain isotopes improving, previously unimportant or unknown background contributions must be understood and eliminated. To that end, the contribution from long-lived isotopes produced by cosmic-ray neutrons in detector and shielding materials must be considered. For example, the work of Ref. [10] measured cosmic activation of ${ }^{76} \mathrm{Ge}$. Several $0 \nu \beta \beta$ experiments utilize large amounts of low background $\mathrm{Pb}$ as a means to passively shield external radiation. If the $\mathrm{Pb}$ shielding resides on the Earth's surface prior to deployment at an underground laboratory, cosmic activation occurs. No measurements of cosmic activation in $\mathrm{Pb}$ exists or lines identified as such by low background experiments. It is not clear if the lack of prior observations is due to short surface exposures of low background $\mathrm{Pb}$ or due to a small activation rate. Therefore, it is important to understand the production of long-lived activated products in $\mathrm{Pb}$ for future $0 \nu \beta \beta$ decay experiments

We exposed a $\mathrm{Pb}$ foil sample to a broad-energy neutron beam with an energy spectrum similar to the cosmic-ray neutron flux on the Earth's surface. After neutron exposure, the sample is counted on a low-background $\gamma$-ray spectrometer to detect the the decays of produced isotopes. From these data we measure the production rate of activated products due to fast neutrons in the $\mathrm{Pb}$ sample. Combining with the neutron-beam and cosmicneutron energy spectra, we make an estimate of the cosmic ray production of long-lived isotopes in $\mathrm{Pb}$. The measured production rate is compared to a production rate from a cross-section calculation over the appropriate energy range and cosmic-ray neutron flux measurements. This article describes our determination of the production rate of these isotopes. 


\section{Experiment}

The natural $\mathrm{Pb}$ sample was placed in the Weapons Neutron Research (WNR) facility Target 4 Flight Path 60 Right (4FP60R) [11] neutron beam at the Los Alamos Neutron Science Center (LANSCE). The broad-spectrum, pulsed neutron beam at this flight path strikes the $\mathrm{Pb}$ target produce outgoing $\gamma$ rays to interact with the GErmanium Array for Neutron Induced Excitations (GEANIE) spectrometer [12]. The GEANIE spectrometer data and resulting $\left(n, n^{\prime} \gamma\right)$ analysis are presented in a separate publication [13]. The GEANIE sample is located a distance of $20.34 \mathrm{~m}$ from the natural tungsten spallation target.

The neutron target at the center of GEANIE was five stacked foils of natural $\mathrm{Pb}\left({ }^{\text {nat }} \mathrm{Pb}\right)$ angled $20^{\circ}$ from the normal to the beam direction. The neutron beam had a diameter of $1.905 \mathrm{~cm}$. Each foil measured nominally $5 \mathrm{~cm} \times 5 \mathrm{~cm}$ in area and $0.475 \mathrm{~mm}$ in thickness. The Pb sample obtained from GoodFellow has a purity of $99.95 \%$. Typical impurities for this sample include 20 ppm Ag, 100 ppm Bi, 2 ppm Cd, 20 ppm Cu, 2 ppm Fe, < 1 ppm $\mathrm{Mg}, 70 \mathrm{ppm} \mathrm{Sn}$, and $5 \mathrm{ppm} \mathrm{Tl}$.

The neutron exposure occurred over two periods with 2 foils exposed in the year 2003 and all 5 foils later in year 2006. The first 2-foil exposure was performed between Aug. 18 and Aug. 22, 2003 (3.72 days). The full 5-foil exposure occurred between Oct. 13 and Oct. 30, 2006 (16.70 d), Nov. 9 and Nov. 13, 2006 (3.66 d), and on Nov. 22 (0.33 d). The neutron beam is pulsed allowing a measurement of the neutron energy and flux by time of flight with an in-beam fission chamber containing ${ }^{238} \mathrm{U}$ foils [14]. As seen in Fig. 1, the neutron energy spectrum at 4FP60R in 2006 shows good spectral agreement over an energy range between 20-300 MeV with the Gordon [15] parameterization of the sea level cosmic-neutron energy spectra.

For convenience, we provide a parametrization of the neutron spectrum impinging our $\mathrm{Pb}$ target should an alternate cross section or cosmic neutron flux be investigated. The spectrum can be described as:

$$
\begin{aligned}
\Phi(E)= & \left(1.832 \times 10^{10}\right) \times \\
& e^{\left(5.166 \ln E-3.815 \ln ^{2} E+0.895 \ln ^{3} E-0.071 \ln ^{4} E\right)}
\end{aligned}
$$

where $\Phi$ is in units of neutrons/MeV and the energy (E) is in $\mathrm{MeV}$.

The TALYS [16] nuclear reaction code calculates excitation functions for

producing nuclides in $\mathrm{Pb}$. Of the nuclides predicted by TALYS, only ${ }^{194} \mathrm{Hg}$ 


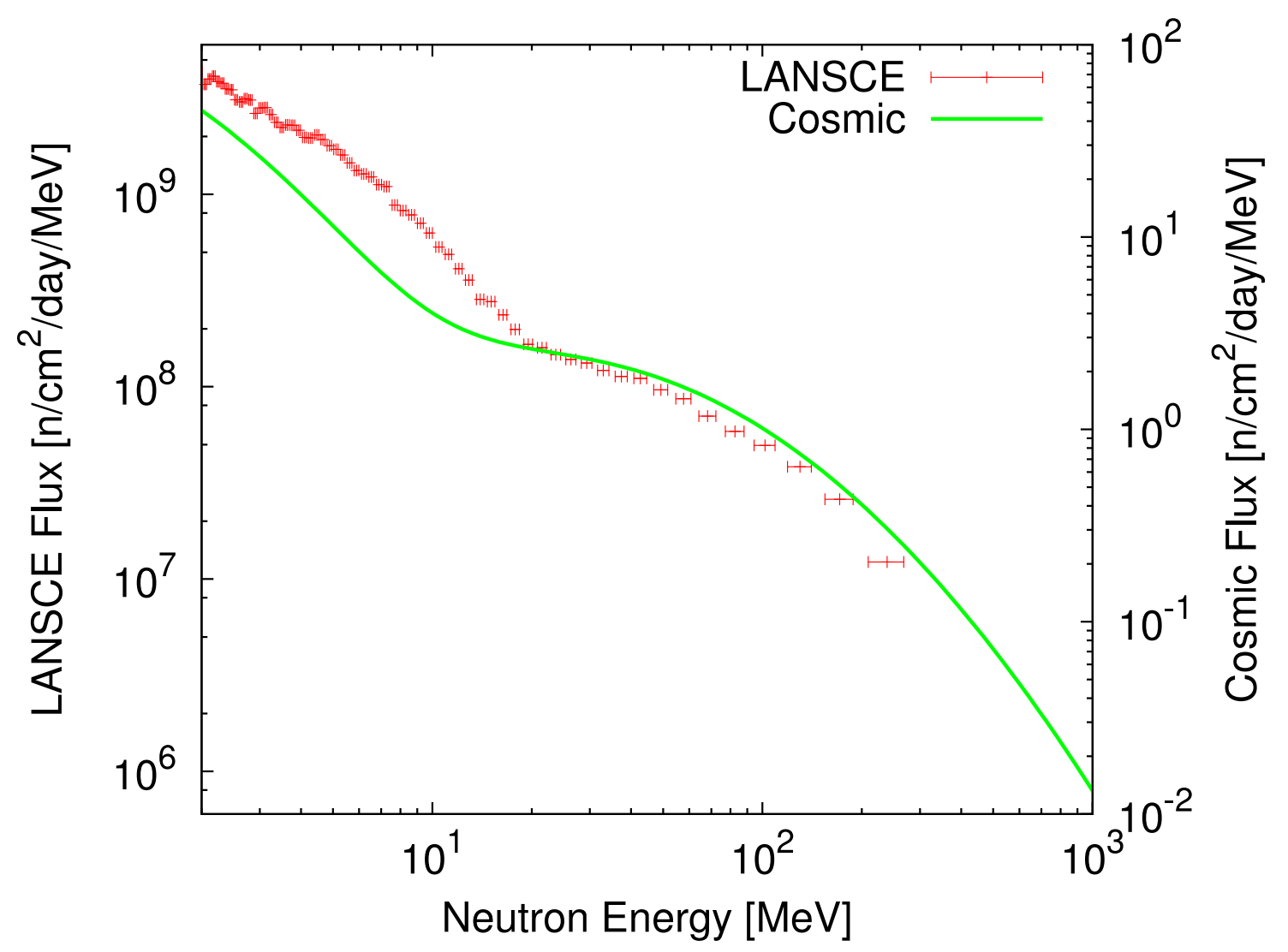

Figure 1: The energy spectrum of the neutron beam at 4FP60R for the 2006 exposure periods. The solid curve represents the sea level cosmic-neutron flux from Ref. [15]. 


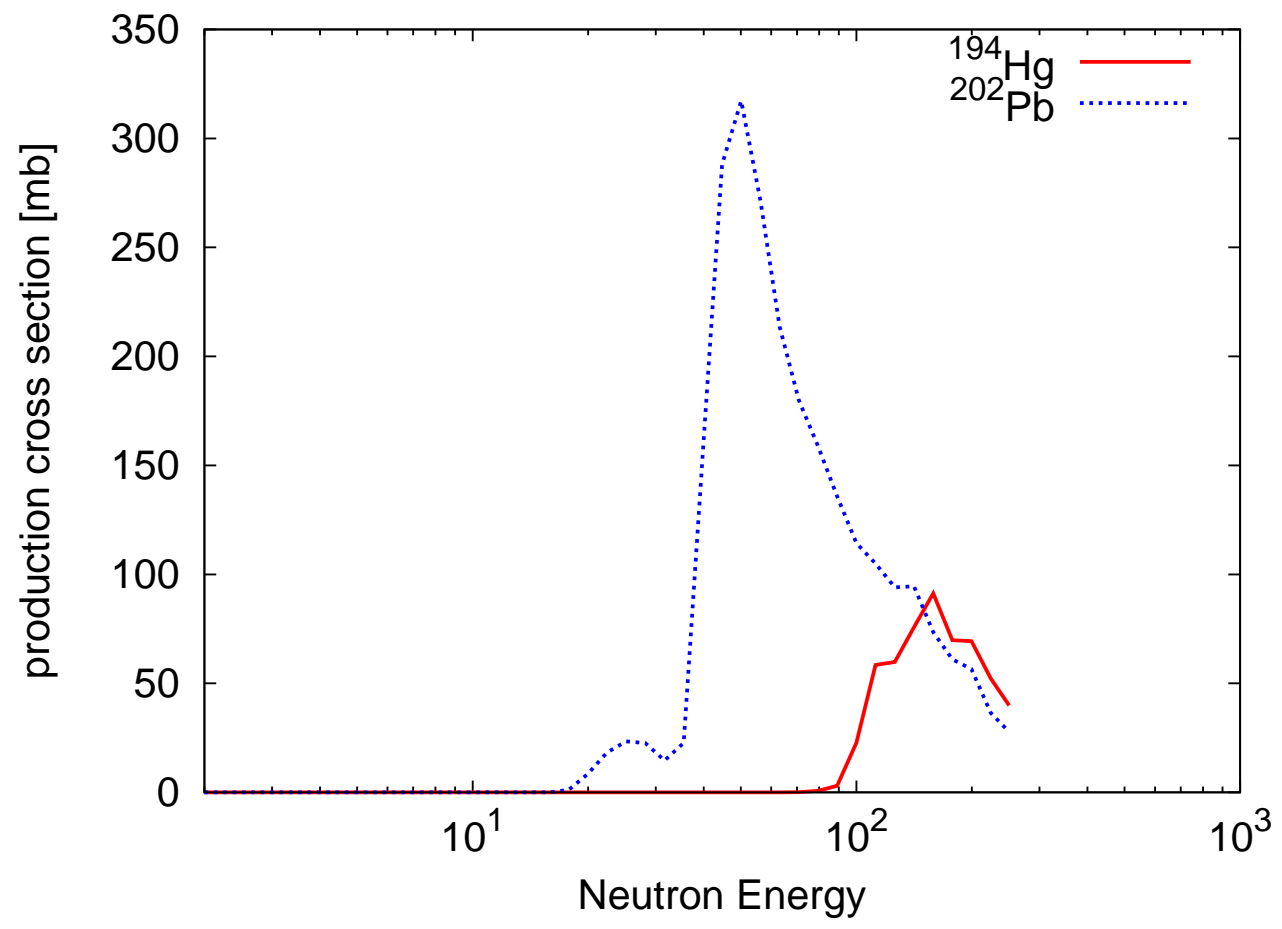

Figure 2: The production cross sections for ${ }^{202} \mathrm{~Pb}$ (top curve) and ${ }^{194} \mathrm{Hg}$ (lower curve) calculated by TALYS for neutrons impinging on natural $\mathrm{Pb}$. The neutron energy range of TALYS is limited to $250 \mathrm{MeV}$.

and ${ }^{202} \mathrm{~Pb}$ are long-lived $\left(t_{1 / 2}>1 \mathrm{yr}\right)$ and support radioisotopes with a decay mode to an excited state so that $\gamma$-ray emission is possible. ${ }^{194} \mathrm{Hg}$ supports the shorter lived ${ }^{194} \mathrm{Au}\left(t_{1 / 2}=38.0 \mathrm{~h}\right)$ and ${ }^{202} \mathrm{~Pb}$ supports the shorter lived ${ }^{202} \mathrm{Tl}\left(t_{1 / 2}=12.3 \mathrm{~d}\right)$ [17]. The production cross sections for these two nuclides are shown in Fig. 2. The production cross section of ${ }^{194} \mathrm{Hg}$ includes feeding from the short-lived nuclides ${ }^{194} \mathrm{Tl}$ and ${ }^{194} \mathrm{~Pb}$. Note that the cross sections are peaked within the energy range of $20-300 \mathrm{MeV}$, which is the same energy region where there is agreement in the shape of the neutron flux between sea level cosmic neutrons and the 4FP60R neutrons (Fig. 1).

The experimental setup at GEANIE is optimized for measuring prompt $\gamma$ rays due to neutron reactions in a target sample. During periods of time while the data acquisition system is offline, neutron data is not collected by the in-beam fission chamber. Therefore, a direct measurement of the total 
neutron yield on target is not possible. Instead, we rely on an upstream proton current monitor that continuously logs the beam current directed at WNR spallation target. This monitor provides a better handle on the total neutron current delivered to the flight path if the fission chamber neutron monitor experiences periods of downtime (e.g. in between data runs, during DAQ malfunctions and debugging, etc.). A subset of the irradiation period while the neutron fission chamber is online can be used to normalize the proton current to delivered neutron current.

After irradiation, the $\mathrm{Pb}$ foils were stored for an extended period and therefore any radioactivity had decayed to an extremely low level $(<10 \mathrm{~Bq})$ before counting began. Therefore, the activity of the sample was negligible to sample handling and transportation requirements. The sample was taken to our low background High Purity Germanium (HPGe) underground counting facility at the Waste Isolation Pilot Plant (WIPP) near Carlsbad, NM. The HPGe detector and shielding have been underground at WIPP since 1998. This n-type semi-coax detector has a height of $41 \mathrm{~mm}$ and a diameter of $51 \mathrm{~mm}$. The cryostat is made from $\approx 1-\mathrm{mm}$ thick $\mathrm{Cu}$ cryostat. The shield comprises $5 \mathrm{~cm}$ of oxygen-free, high-conductivity $\mathrm{Cu}$ and $10 \mathrm{~cm}$ of $\mathrm{Pb}$.

The sample was counted over a period of 126.6 days between Aug. 4 and Dec. 8, 2010 with a break in data collection resulting in a total live time of 109.07 days. The $\gamma$-ray energy spectrum is shown in Fig. 3. Three lines from ${ }^{194} \mathrm{Au}$, a line from ${ }^{202} \mathrm{Tl}$, and two lines from ${ }^{207} \mathrm{Bi}$ are observed in the activated spectra in addition to background lines. The $\gamma$-ray detection efficiency is determined by Monte Carlo simulations using the GEANT4based MaGe framework [18]. The simulated geometry includes five sheets of $\mathrm{Pb}$ placed on the HPGe detector. The simulated $\gamma$ rays originate uniformly throughout a cylindrical section of the $\mathrm{Pb}$ sheet consistent with the beam spot diameter. Therefore, the $\gamma$-ray detection efficiency also takes into account self shielding of the $\mathrm{Pb}$ foils in addition to the shielding of the HPGe cryostat and the detector response.

\section{Measured Production Rates}

The observed peaks in the $\gamma$-ray spectrum (Fig. 3) are fit to determine the measured counts $(C)$ that are tabulated in Table 1. The measured energy spectrum shows evidence of the expected long-lived activated isotopes. In some of the peaks, the same lines are observed in the background spectra. In the case of ${ }^{207} \mathrm{Bi}$, the $570-\mathrm{keV}$ and $1064-\mathrm{keV} \gamma$ ray lines are also 

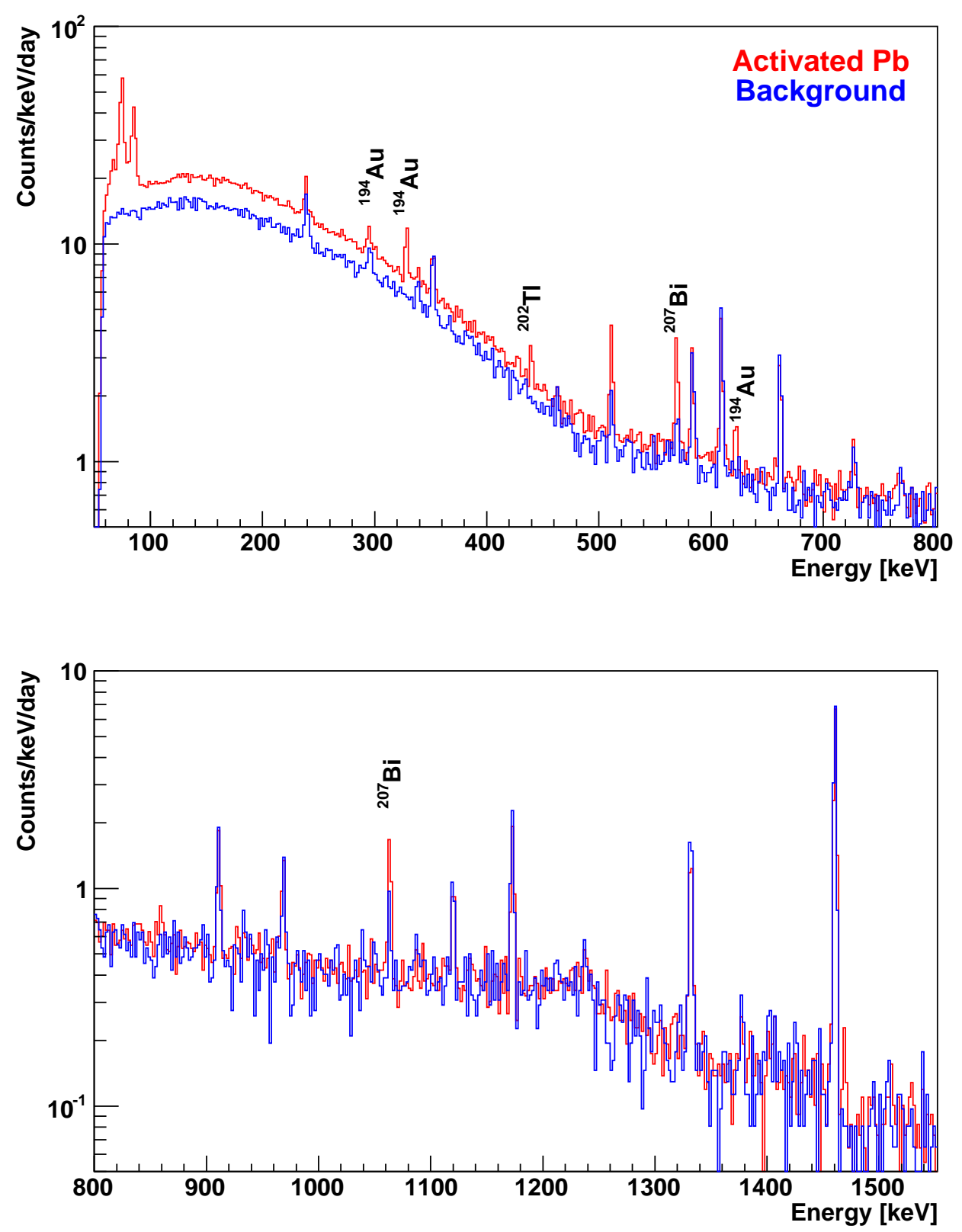

Figure 3: The energy spectrum of $\gamma$ rays from the $\mathrm{Pb}$ sample (red) as measured by a HPGe detector and a background spectrum (blue) taken with no sample present. 
found in the background spectra at a lower rate. The $294-\mathrm{keV} \gamma$-ray line attributed to ${ }^{194} \mathrm{Au}$ is also found in the background spectra due to naturally occurring ${ }^{214} \mathrm{~Pb}$. The measured counts in Table 1 represent the net counts after background subtraction for these three lines. Note that the $329-\mathrm{keV}$ line could be due to decay of naturally occurring ${ }^{228}$ Ac. However, the background spectrum shows no evidence of this ${ }^{228} \mathrm{Ac}$ line and no other ${ }^{228} \mathrm{Ac}$ lines are observed in the sample spectrum. Therefore the entire $328-\mathrm{keV}$ line is attributed to the decay of ${ }^{194} \mathrm{Au}$.

The $\gamma$-ray detection efficiency $\left(\epsilon_{\gamma}\right)$ was determined by Monte Carlo simulations benchmarked against measurements of calibrated radioactive standards $\left({ }^{57,60} \mathrm{Co},{ }^{54} \mathrm{Mn},{ }^{22} \mathrm{Na}\right.$ and $\left.{ }^{137} \mathrm{Cs}\right)$. We use a random pulser to verify the event-rate dependence of the data-acquisition system dead time.

In addition to $\epsilon_{\gamma}$, an additional correction factor accounts for the decay of the isotope since the end of exposure and during the counting period. This latter correction factor $\left(\epsilon_{c}\right)$ listed in Table 1 depends on the half-life of the isotope of interest.

It is straight-forward to calculate the number of atoms of each isotope that were present at the end of neutron exposure (November 22, 2006) and the production rate. The measured number of counts $(C)$ is related to the number of atoms $\left(N_{i}\right)$ on the reference date at the end of exposure by:

$$
C=\epsilon_{c} \sum_{i} N_{i} \epsilon_{\gamma_{i}}
$$

where the sum is over the four irradiation periods. Since the first irradiation period only included two of the $\mathrm{Pb}$ foils, atoms produced from that run have a different $\gamma$-ray detection efficiency than those produced in the latter three runs with all five $\mathrm{Pb}$ foils. The $\gamma$-ray counting occurred over two periods of time with a short break in between. The decay of the isotope before and during the two counting periods is corrected with a sum over the two periods by

$$
\epsilon_{c}=\sum_{j}\left(e^{-\lambda T_{j}^{s t a r t}}-e^{-\lambda T_{j}^{e n d}}\right),
$$

where $T_{j}^{e n d}\left(T_{j}^{\text {start }}\right)$ is the number of days since the end of exposure that the counting stopped (started) for each of the $j$ counting periods, and $\lambda$ is the decay constant of the isotope in question. 


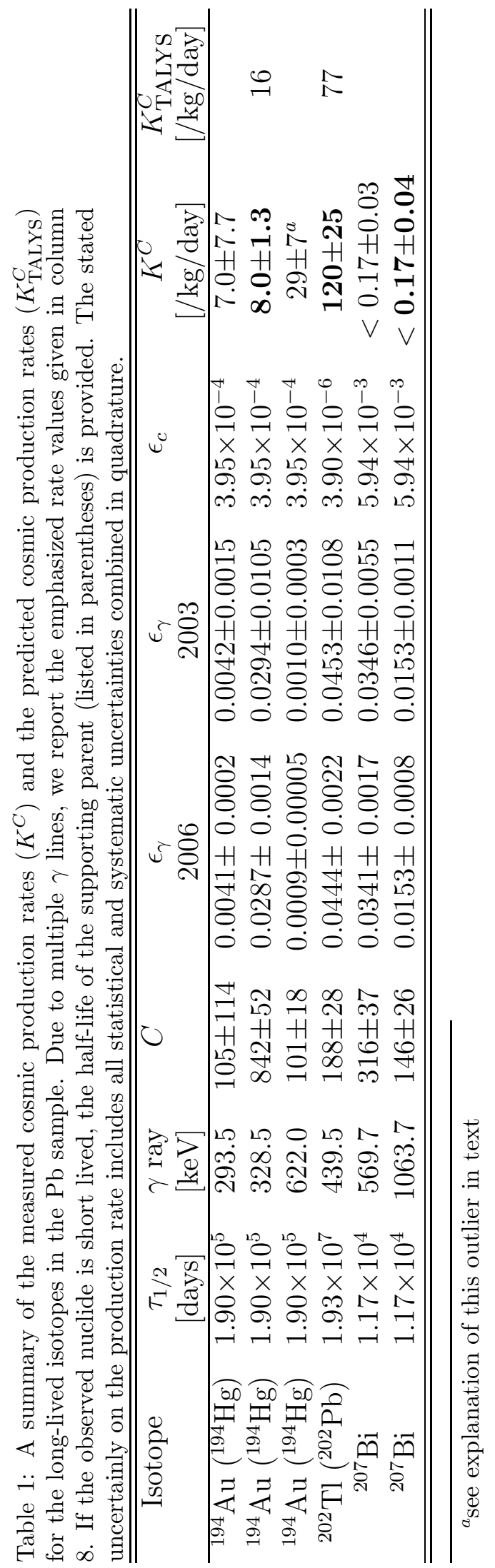


The number of atoms can also be expressed in terms of the reaction rates during an exposure period:

$$
N_{i}=\frac{K_{i}^{L} M_{i}}{\lambda}\left(1-e^{-\lambda T_{i}^{i r r a d}}\right) e^{-\lambda T_{i}^{\text {decay }}}
$$

where we have corrected for the decay during the exposure and the decay after the exposure until the reference date. In Eqn. 4, $T_{i}^{\text {irrad }}$ is the duration of irradiation of the $i^{\text {th }}$ exposure, and $T_{i}^{\text {decay }}$ is the time between the end of exposure $i$ and the reference date. $K_{i}^{L}$ is the production rate [atoms $/ \mathrm{kg} /$ day] during the LANSCE exposure $i$, and $M_{i}$ is the mass of the sample within the beam spot.

The production rate of the identified long-lived nuclides in $\mathrm{Pb}$ occurs in an energy range where the shapes of the LANSCE and cosmic neutron fluxes are similar. Hence, a simple scaling of the LANSCE production rate is sufficient to estimate the cosmic production rate, given by

$$
\begin{aligned}
K^{C} & =K_{i}^{L} \frac{f^{C}}{f_{i}^{L}} \\
& =K_{i}^{L} \frac{f^{C}}{S_{i} I_{i} / A_{i} / T_{i}^{\text {irrad }}}
\end{aligned}
$$

where $K^{C}$ is the cosmic production rate [atoms $/ \mathrm{kg} /$ day], $f^{C}\left(f^{L}\right)$ is the energy-integrated neutron flux $\left[\mathrm{n} / \mathrm{cm}^{2} /\right.$ day] for cosmic (LANSCE) neutrons over the mutual energy range $20-300 \mathrm{MeV}, S_{i}$ is the normalization between proton current and neutron current, and $A_{i}$ is the cross-sectional area of the $\mathrm{Pb}$ target in the neutron beam. The sea-level cosmic neutron flux of Ref. [15] is assumed where $f^{C}=228 \mathrm{n} / \mathrm{cm}^{2} /$ day.

Eqn. 4 can be written as

$$
C=\frac{K^{C} \epsilon_{c}}{f^{C} \lambda} \sum_{i} \frac{n_{i} S_{i} I_{i}}{T_{i r r a d}^{i}} \epsilon_{\gamma_{i}}\left(1-e^{-\lambda T_{i}^{i r r a d}}\right) e^{-\lambda T_{i}^{d e c a y}}
$$

where the $M_{i} / A_{i}$ term is replaced by the areal density $\left(n_{i}\right)$ of the $\mathrm{Pb}$ target parallel to the beam direction. The beam spot covers most, but not all, of the sample geometry.

Table 1 lists the isotopes found and the number of measured counts after irradiations. Eqn. 7 allows a direct determination of the cosmic production rate $K^{C}$, also listed in Table 1 . Also listed is the cosmic activation rate 
calculated with the TALYS excitation functions (Fig. 2 and the sea level cosmic neutrons [15]).

The production of long-lived ${ }^{194} \mathrm{Hg}$ is found through the presence of characteristic $\gamma$ rays from supported progeny ${ }^{194} \mathrm{Au}$. The production rates found by the three ${ }^{194} \mathrm{Au}$ do not all agree: The $294-\mathrm{keV}$ and $329-\mathrm{keV}$ lines agree while that found by the $622-\mathrm{keV}$ line is nearly a factor of four higher. Of the three lines, two have low branching ratios and so we place the most confidence on the stronger $329-\mathrm{keV}$ line. Further, the $622-\mathrm{keV}$ line can occur as a lone $\gamma$ ray or as a sum of the $293.5-\mathrm{keV}$ and $328.5-\mathrm{keV} \gamma$ rays. The Monte Carlo of the $\gamma$-ray detection efficiency is based on the decay of ${ }^{194} \mathrm{Au}$ and hence the cascade is modeled in the efficiency calculation and likely not the sole cause of the observed excess. There are at least four other $\gamma$-ray lines expected from ${ }^{194} \mathrm{Au}$ in the observed energy range with non-negligible intensities: $364.8 \mathrm{keV}, 482.8 \mathrm{keV}, 528.8 \mathrm{keV}, 645.2 \mathrm{keV}$. No peaks are observed at these energies and the background level is used to set production rate limits (90\% confidence level) of $17(3), 11(2), 9(1)$, and $7(1)$ atoms $/ \mathrm{kg} /$ day. These limits rule out the larger production rate found by the $622-\mathrm{keV}$ line. Its excess may be due to a blend from another source at the same energy. One likely candidate is the $622-\mathrm{keV} \gamma$ ray from ${ }^{106} \mathrm{Rh}$, which is progeny of fission product ${ }^{106} \mathrm{Ru}$ predicted by TALYS to be activated at a rate of 0.05 atoms $/ \mathrm{kg} /$ day. If we assume the excess of the $622-\mathrm{keV}$ line is due to ${ }^{106} \mathrm{Rh}$, we find a cosmic activation rate of $0.18 \pm 0.06$ atoms $/ \mathrm{kg} /$ day for that isotope.

The production of ${ }^{202} \mathrm{~Pb}$ is observed through the lone $\gamma$ ray from ${ }^{202} \mathrm{Tl}$. We mentioned above that the irradiated $\mathrm{Pb}$ sample may have impurities, which could also be targets for neutron activation. Of the listed impurities, only Bi provides an additional means to activate ${ }^{202} \mathrm{~Pb}$ and ${ }^{194} \mathrm{Au}$. A TALYS calculation of the production in $\mathrm{Bi}$ at the stated impurity levels is found to be negligible compared the the rate found in natural $\mathrm{Pb}$.

While the production of ${ }^{207} \mathrm{Bi}$ in natural $\mathrm{Pb}$ is observed, some comments on its measured production are necessary. First, the Bi impurity in the natural $\mathrm{Pb}$ sample is large enough to have a significant effect on the production of ${ }^{207} \mathrm{Bi}$. A TALYS calculation finds the production of ${ }^{207} \mathrm{Bi}$ in $100 \mathrm{ppm}$ Bi to be $10 \%$ of that measured in natural $\mathrm{Pb}$. Therefore, the measured production rate may be partly due to the $\mathrm{Bi}$ impurity of the sample and therefore we report our result as an upper limit only. Second, Bi has a higher atomic number than $\mathrm{Pb}$ and hence the reaction requires an incoming proton. It is likely that $\mathrm{Bi}$ is produced from a secondary reaction from residual protons left from incident neutrons. This secondary production is not directly pre- 
dicted by TALYS. To quantify the likelihood of secondary reactions involving proton capture, a two-step TALYS calculation is made. The first predicts the proton flux from residual protons emitted from incident cosmic neutrons on $\mathrm{Pb}$. Next, the proton flux generates secondary reactions on $\mathrm{Pb}$ that produce ${ }^{207} \mathrm{Bi}$ at a rate of 0.04 atoms $/ \mathrm{kg} /$ day, which is a factor of 5 less than measured here. Therefore, its plausible that the suspected secondary proton capture reactions causes the ${ }^{207} \mathrm{Bi}$ production found up the rate reported.

The aerial densities and total neutron yield for the two irradiation periods are listed in Table 2. The low neutron yield and lower $\mathrm{Pb}$ thickness in the 2003 run make it a small contribution to the total production of nuclides.

Table 2: A summary of the total neutron yield for the two irradiation years.

\begin{tabular}{ccc}
\hline \hline Year & $\begin{array}{c}n \\
{\left[\mathrm{~g} / \mathrm{cm}^{2}\right]}\end{array}$ & $\begin{array}{c}\text { Neutrons on target }(\mathrm{S} \times \mathrm{I}) \\
\text { between } 20-300 \mathrm{MeV}\end{array}$ \\
\hline 2003 & 1.215 & $5.73 \pm 5.46 \times 10^{10}$ \\
2006 & 3.038 & $6.77 \pm 0.28 \times 10^{11}$ \\
\hline \hline
\end{tabular}

The uncertainty in the production rate is dominated by the counting statistics of weak peaks (Table 1), the counting efficiency, the exposed neutron yield, and the cosmic ray neutron flux. The $\gamma$-ray efficiency for the full five $\mathrm{Pb}$ foils exposed in 2006 is known to $5 \%$, which is based on source data and Monte Carlo agreement. The placement of the two $\mathrm{Pb}$ foils exposed in 2003 within the five foils during $\gamma$ counting is unknown, though they were adjacent. Therefore, the $2003 \gamma$-ray detection efficiency has a larger systematic uncertainty (Table 1). The uncertainty on neutron yield is due to the statistical uncertainty on the proton beam current and the uncertainty on the proton to neutron normalization. The 2006 total neutron yield is known to $4.2 \%$. For the 2003 neutron yield calculation, the limited fission chamber data available suggests a lower proton to neutron normalization, though no physical explanation can explain the discrepancy with the 2006 normalization. We assume the nominal 2006 normalization for the 2003 neutron yield, but conservatively include a larger uncertainty of $95 \%$ required to account for the difference found when using the limited fission chamber data. The 2003 exposure represents $3.3 \%$ of the total neutron exposure so the large uncertainty on the 2003 neutron yield has a small effect on the net production rate. 
The precision to which the cosmic ray neutron flux is measured is claimed to be within $10-15 \%$ [15] and so we assume an uncertainty of $12.5 \%$. The total cosmic flux also includes contributions from subdominant proton and pion interactions. This neglected contribution is approximately $10 \%$ [19] of the total production rate. These charged particles are much less penetrating than neutrons and therefore their impact on any given sample is very geometry dependent. Hence we assume a $50 \%$ uncertainly on this correction for an effective uncertainty of $5 \%$ on the neglected contribution. Since the two contributes to the cosmic uncertainty are uncorrelated, we use a net estimated systematic uncertainty on the cosmic neutron flux of $13.5 \%$. The counting durations live times are known to a small percentage and are negligible contributions to the uncertainty. This certainty is also true for the irradiation times. The sample had been stored on the Earth's surface for many years prior to exposure to the neutron beam. Any isotopes produced by cosmic ray neutrons would be below saturation after this extended period is ignored given a cosmic contribution $\ll 3 \%$ than that produced by the intense neutron beam. The half-lives and branching ratios of the produced isotopes are known to high precision and are a negligible contribution to the total uncertainty.

\section{Discussion}

Previous low background experiments using $\mathrm{Pb}$ shielding have not found lines in their detector's energy spectrum attributed to long-lived activated products in $\mathrm{Pb}$. For example, the HEILDELBERG-MOSCOW (H-M) [20] neutrinoless double-beta decay experiment does not present evidence of ${ }^{194} \mathrm{Au}$ or ${ }^{202} \mathrm{Tl}$. The H-M spectra does show the presence of ${ }^{207} \mathrm{Bi}$, though its attributed to anthropogenic rather that cosmic production in $\mathrm{Pb}$ shielding. The ${ }^{207} \mathrm{Bi}$ may be partially cosmogenic in origin in the $\mathrm{Pb}$ shielding. If cosmogenic, the decay rate of ${ }^{207} \mathrm{Bi}$ observed here suggests that ${ }^{194} \mathrm{Au}$ and ${ }^{202} \mathrm{Tl}$ would also be observed. However, the activation of ${ }^{207} \mathrm{Bi}$ here is due to secondary reactions involving protons and the net cosmic production would be greater due to the flux of cosmic protons.

The work of Mei et al. [21] using a Pb shield surrounding a HPGe detector did observe an unidentified line at $438.9 \mathrm{keV}$, which could be attributed to the ${ }^{202} \mathrm{Tl}$ line at $439.5 \mathrm{keV}$. However, a long term spectra obtained by Ref. [22] also observed a line at $438.8 \mathrm{keV}$ but attributed it to a ${ }^{40} \mathrm{~K}$ double escape peak. The relative likelihood of this line being attributed to either 
cosmogenic ${ }^{202} \mathrm{~Pb}$ or ${ }^{40} \mathrm{~K}$ should be further investigated. If cosmogenics in $\mathrm{Pb}$ are present, its more likely that the more abundant ${ }^{194} \mathrm{Au}$ would be present. The two experiments see the $329-\mathrm{keV}$ line attributed to ${ }^{228} \mathrm{Ac}$, which could be blending with cosmogenic ${ }^{194} \mathrm{Au}$. Instead, the rate of the $329-\mathrm{keV}$ line is consistent with the nearby $338-\mathrm{keV}$ line from ${ }^{228} \mathrm{Ac}$ when scaled by their branching ratios suggesting little evidence for the cosmogenic ${ }^{194} \mathrm{Au}$.

Neutrinoless double-beta decay experiments typically choose target nuclei with a high $(\geq 2 \mathrm{MeV})$ double-beta $\mathrm{Q}$ value in order to stay above most $\gamma$ rays from natural sources. The activated products discovered here do pose a potential background contribution due to their high decay $\mathrm{Q}$ values and high energy $\gamma$ rays. While ${ }^{194} \mathrm{Au}$ has a decay $\mathrm{Q}$-value of $2501 \mathrm{keV}$ with $\gamma$ rays up to $2413 \mathrm{keV}$ and ${ }^{207} \mathrm{Bi}$ has a decay Q-value of $2398 \mathrm{keV}$ with $\gamma$ rays up to $1770 \mathrm{keV},{ }^{202} \mathrm{Tl}$ is less of a concern with a lower Q value of $1363 \mathrm{keV}$ [17]. The production rates of these cosmically activated radioisotopes in $\mathrm{Pb}$ can be used to estimate background contributions and surface exposure limitations for the next generation neutrinoless double-beta decay experiments.

With its high energy $\gamma$ rays, ${ }^{194} \mathrm{Au}$ is likely the most problematic cosmogenic in $\mathrm{Pb}$ shielding. The highest intensity $\gamma$-ray line above $2 \mathrm{MeV}$ occurs at $2043.7 \mathrm{keV}$. This transition is close to the $2039-\mathrm{keV}$ Q value of double-beta decay target ${ }^{76} \mathrm{Ge}$. Using the production rate measured here, cosmogenic ${ }^{194} \mathrm{Au}$ will saturate at a concentration of $93 \mu \mathrm{Bq} / \mathrm{kg}$. With a decay intensity of $3.81 \%$, the $2044-\mathrm{keV} \gamma$ ray would be produced at a rate of $0.0035 \mathrm{mHz} / \mathrm{kg}$ at saturation. As stated earlier, the $\mathrm{H}-\mathrm{M}$ experiment did not see evidence of ${ }^{194} \mathrm{Au}$. To estimate their sensitivity to a $2 \mathrm{MeV} \gamma$ ray, they did observe the $2.6-\mathrm{MeV} \gamma$ ray from ${ }^{208} \mathrm{Tl}$ in the ${ }^{232} \mathrm{Th}$ decay chain and determined the concentration to be $12.3 \mu \mathrm{Bq} / \mathrm{kg}$ of ${ }^{232} \mathrm{Th}\left(4 \mu \mathrm{Bq} / \mathrm{kg}\right.$ of $\left.{ }^{208} \mathrm{Tl}\right)$ in the Pb shielding [23]. Therefore, they would have sensitivity to ${ }^{194} \mathrm{Au}$ at saturation indicating the $\mathrm{Pb}$ shielding had little exposure to cosmic neutrons at the surface of the Earth prior to use underground. Current and future experiments are finding shielding materials with improved contamination levels. $\mathrm{Pb}$ suitable for shielding has been obtained at $<4 \mu \mathrm{Bq} / \mathrm{kg}$ of ${ }^{232} \mathrm{Th}\left(<1.3 \mu \mathrm{Bq} / \mathrm{kg}\right.$ of $\left.{ }^{208} \mathrm{Tl}\right)$ [24]. Since ${ }^{232} \mathrm{Th}$ contamination in $\mathrm{Pb}$ is currently unavoidable, it can be used to set the scale of other contaminate goals. In order to keep the decay rate of the $2044-\mathrm{keV} \gamma$ ray at or below the decay rate of the ${ }^{208} \mathrm{Tl} 2614-\mathrm{keV}$ $\gamma$ ray, $\mathrm{Pb}$ surface exposure should be limited to $350 \mathrm{yr}$. A limitation of 30 yr of surface exposure is required to stay a factor of 10 less than the ${ }^{208} \mathrm{Tl}$ decay rate. 


\section{Conclusion}

We measure the production of ${ }^{194} \mathrm{Hg},{ }^{202} \mathrm{~Pb}$, and ${ }^{207} \mathrm{Bi}$ in a sample of $\mathrm{Pb}$ due to high-energy neutron interactions within a neutron beam with a spectrum similar to that of the cosmic-ray neutron flux at the Earth's surface. These results are used to predict sea level neutron activation of long-lived nuclides in $\mathrm{Pb}$ shielding. Measurements of $\gamma$-ray emissions reveal the presence of long-lived isotopes and we set production rates of $8.0 \pm 1.3$ atoms $/ \mathrm{kg} /$ day of

${ }^{194} \mathrm{Hg}, 120 \pm 25$ atoms $/ \mathrm{kg} /$ day ${ }^{202} \mathrm{~Pb}$, and $<0.17 \pm 0.04$ atoms $/ \mathrm{kg} /$ day ${ }^{207} \mathrm{Bi}$. Neutron activation of natural $\mathrm{Pb}$ can be a source of background for rare event searches such as neutrinoless double-beta decay experiments. Based on previous low background $\gamma$ ray spectrometers using $\mathrm{Pb}$ shielding and given the production rates measured here, we can estimate the surface exposure necessary for neutron-activated products to be a significant background source. The cosmogenic background would require a sea level exposure of $350 \mathrm{yr}$ to be commensurate with the background from ${ }^{208} \mathrm{Tl}$ in ultra-pure $\mathrm{Pb}$ shielding. A shorter exposer would guarantee the cosmogenic background is less than the naturally occurring radioisotopes in $\mathrm{Pb}$. Therefore, it is not strictly necessary to provide ultra-pure $\mathrm{Pb}$ shielding short-term protection from cosmic rays in the execution of low background, rare event searches using $\mathrm{Pb}$.

\section{Acknowledgments}

This material is based upon work supported by the U.S. Department of Energy, Office of Science, Office of Nuclear Physics under Award Number DE-SCOO05054, the LANL LDRD Program, and the DOE/NNSA Stewardship Science Graduate Fellowship Program under grant number DE-FC5208NA28752. This work benefited from the use of the Los Alamos Neutron Science Center, funded by the U.S. Department of Energy under contract DE-AC52-06NA25396. This work also benefited from our underground laboratory at the Waste Isolation Pilot Plant (WIPP), which we operate with support from the Nuclear Physics office of the U.S. Department of Energy under contract number 2011LANLE9BW. Finally, we thank our friends and hosts at the Waste Isolation Pilot Plant (WIPP) for their continuing support of our activities underground at that facility. 


\section{References}

[1] S. R. Elliott, P. Vogel, Double beta decay, Ann. Rev. Nucl. Part. Sci. $52(2002) 115$.

[2] S. R. Elliott, J. Engel, Double-beta decay, J. Phys. G: Nucl. Part. Phys. 30 (2004) R183-R215.

[3] F. T. Avignone III, S. R. Elliott, J. Engel, Double beta decay, Majorana neutrinos, and neutrino mass, Rev. Mod. Phys. 80 (2008) 481-516.

[4] W. Rodejohann, Neutrino-less Double Beta Decay and Particle Physics, Int. J. Mod. Phys. E 20 (2011) 1833.

[5] A. Barabash, Double beta decay experiments, Physics of Particles and Nuclei 42 (2011) 613-627.

[6] J. J. Gomez-Cadenas, J. Martin-Albo, M. Mezzetto, F. Monrabal, M. Sorel, The search for neutrinoless double beta decay, Riv. Nuovo Cim. 35 (2012) 29-28.

[7] S. R. Elliott, Recent Progress in Double Beta Decay, Mod. Phys. Lett. A 27 (2012) 1230009.

[8] M. Auger, et al., Search for Neutrinoless Double-Beta Decay in ${ }^{136} \mathrm{Xe}$ with EXO-200, Phys. Rev. Lett. 109 (2012) 032505.

[9] S. Agostini, et al., Results on neutrinoless double beta decay of ${ }^{76} \mathrm{Ge}$ from Gerda Phase I, Phys. Rev. Lett. 111 (2013) 122503.

[10] S. R. Elliott, V. E. Guiseppe, R. A. Johnson, B. H. LaRoque, S. G. Mashnik, Fast-neutron activation of long-lived isotopes in enriched Ge, Phys. Rev. C 82 (2010) 054610.

[11] P. W. Lisowski, C. D. Bowman, G. J. Russel, S. A. Wender, The Los Alamos National Laboratory Spallation Neutron Sources, Nucl. Sci. Eng. 106 (1990) 208.

[12] J. A. Becker, R. O. Nelson, New Opportunities in Nuclear Science with GEANIE at LANSCE/WNR, Nucl. Phys. News Int. 7 (June, 1997) 11. 
[13] V. E. Guiseppe, M. Devlin, S. R. Elliott, N. Fotiades, A. Hime, D.M. Mei, R. O. Nelson, D. V. Perepelitsa, Neutron inelastic scattering and reactions in natural $\mathrm{Pb}$ as a background in neutrinoless double-beta decay experiments, Phys. Rev. C 79 (2009) 054604.

[14] S. A. Wender, et al., A fission ionization detector for neutron flux measurements at a spallation source, Nucl. Instr. Meth. A 336 (1993) 226-231.

[15] M. S. Gordon, P. Goldhagen, K. P. Rodbell, T. H. Zabel, H. H. K. Tang, J. M. Clem, P. Bailey, Measurement of the flux and energy spectrum of cosmic-ray induced neutrons on the ground, IEEE Trans. Nucl. Sci 51 (2004) 3427-3434.

[16] A. J. Koning, S. Hilaire, M. C. Duijvestijn, Talys: Comprehensive nuclear reaction modeling, in: R. C. Haight, M. B. Chadwick, T. Kawano, P. Talou (Eds.), Proceedings of the International Conference on Nuclear Data for Science and Technology - ND2004, AIP vol. 769, Sep. 26 - Oct. 1, 2004, Santa Fe, USA, 2005, p. 1154.

[17] National Nuclear Data Center, http://www.nndc.bnl.gov/, accessed June 2011.

[18] M. Boswell, et al., MaGe-a Geant4-Based Monte Carlo Application Framework for Low-Background Germanium Experiments, IEEE Trans. Nuc. Sci 58 (2011) 1212-1220.

[19] I. Barabanov, S. Belogurov, L. Bezrukov, A. Denisov, V. Kornoukhov, N. Sobolevsky, Cosmogenic activation of Germanium and its reduction for low background experiments, Nucl. Instrum. Meth.B 251 (2006) 115120 .

[20] H. V. Klapdor-Kleingrothaus, et al., Latest Results from the HeidelbergMoscow Double-Beta-Decay Experiment, Eur. Phys. J. A 12 (2001) 147.

[21] D.-M. Mei, S. R. Elliott, A. Hime, V. M. Gehman, K. Kazkaz, Neutron inelastic scattering processes as background for double-beta decay experiments, Phys. Rev. C 77 (2008) 054614.

[22] P. Bossew, A very long-term HPGe-background gamma spectrum, App. Rad. Iso. 62 (2005) 635-644. 
[23] C. Dörr, H. V. Klapdor-Kleingrothaus, New Monte-Carlo simulation of the HEIDELBERG-MOSCOW double beta decay experiment, Nucl. Inst. Meth. A 513 (2003) 596-621.

[24] D. S. Leonard, et al., Systematic study of trace radioactive impurities in candidate construction materials for EXO-200, Nucl. Inst. Meth. A 591 (2008) 490-509. 